\title{
Ultra-low-input, tagmentation-based whole-genome bisulfite sequencing
}

\author{
Andrew Adey and Jay Shendure ${ }^{1}$ \\ Department of Genome Sciences, University of Washington, Seattle, Washington 98195, USA
}

\begin{abstract}
We have adapted transposase-based in vitro shotgun library construction ("tagmentation") for whole-genome bisulfite sequencing. This method, $\mathrm{Tn} 5 \mathrm{mC}$-seq, enables a $>100$-fold reduction in starting material relative to conventional protocols, such that we generate highly complex bisulfite sequencing libraries from as little as $10 \mathrm{ng}$ of input DNA, and ample useful sequences from $1 \mathrm{ng}$ of input DNA. We demonstrate $\mathrm{Tn} 5 \mathrm{mC}$-seq by sequencing the methylome of a human lymphoblastoid cell line to $\sim 8.6 \times$ high-quality coverage of each strand.
\end{abstract}

[Supplemental material is available for this article.]

DNA methylation is a widespread epigenetic modification that plays a pivotal role in the regulation of the genomes of diverse organisms. The most prevalent and widely studied form of DNA methylation in mammalian genomes occurs at the five carbon position of cytosine residues, usually in the context of the CpG dinucleotide. Microarrays, and more recently massively parallel sequencing, have enabled the interrogation of cytosine methylation $(5 \mathrm{mC})$ on a genome-wide scale (Zilberman and Henikoff 2007). However, the in vivo study of DNA methylation and other epigenetic marks, e.g., in specific cell types or anatomical structures, is sharply limited by the relatively high amount of input material required for contemporary protocols.

Methods for genome-scale interrogation of methylation patterns include several that are preceded by the enrichment of defined subsets of the genome (Meissner et al. 2005; Down et al. 2008; Deng et al. 2009), e.g., reduced representation bisulfite sequencing (RRBS) (Meissner et al. 2005) and anti-methylcytosine DNA immunoprecipitation followed by sequencing (MeDIP-seq) (Down et al. 2008). An advantage of such methods is that they can be performed with limited quantities of starting DNA (Gu et al. 2011). However, they are constrained in that they are not truly comprehensive. For example, the digestion-based RRBS method interrogates only $\sim 12 \%$ of CpGs, primarily in CpG islands (Harris et al. 2010), with poor coverage of methylation in gene bodies (Ball et al. 2009) and elsewhere. Furthermore, RRBS does not target cytosines in the $\mathrm{CHG}$ or $\mathrm{CHH}(\mathrm{H}=\mathrm{A}, \mathrm{C}, \mathrm{T})$ contexts, which have been shown to be methylated at elevated levels in the early stages of mammalian development (Lister et al. 2009). While a small proportion of non-CpG methylation sites can be observed using RRBS, they are restricted to regions within or highly proximal to CpGislands (Ziller et al. 2011).

The most comprehensive, highest resolution method for detecting $5 \mathrm{mC}$ is whole-genome bisulfite sequencing (WGBS) (Cokus et al. 2008; Lister et al. 2009; Harris et al. 2010). Treatment of genomic DNA with sodium bisulfite chemically deaminates cytosines much more rapidly than $5 \mathrm{mC}$, preferentially converting them to uracils (Clark et al. 1994). With massively parallel sequencing, these can be detected on a genome-wide scale at singlebase-pair resolution. This approach has revealed complex and

\footnotetext{
${ }^{1}$ Corresponding author.

E-mail shendure@uw.edu.

Article published online before print. Article, supplemental material, and publi-
} cation date are at http://www.genome.org/cgi/doi/10.1101/gr.136242.111. unexpected methylation patterns and variation, particularly in the $\mathrm{CHG}$ and $\mathrm{CHH}$ contexts. Furthermore, as the costs of massively parallel sequencing continue to plummet, WGBS is increasingly affordable. However, a key limitation of WGBS is that the current protocols for library construction are based on ligation chemistry and call for $5 \mu \mathrm{g}$ of genomic DNA as input (Cokus et al. 2008; Lister et al. 2009; Li et al. 2010) which is essentially prohibitive for many samples obtained in vivo.

We recently characterized a transposase-based in vitro shotgun library construction method ("tagmentation") that allows for construction of sequencing libraries from greatly reduced amounts of DNA (Fig. 1A; Adey et al. 2010). Briefly, the method utilizes a hyperactive derivative of the Tn5 transposase loaded with discontinuous synthetic oligonucleotides to simultaneously fragment and append adaptors to genomic DNA. The resulting products are subjected to PCR amplification followed by high-throughput sequencing. The increased efficiency of genomic DNA conversion to viable amplicons and the greatly reduced number of steps allow the construction of low-bias, highly complex libraries from $<50 \mathrm{ng}$ of genomic DNA.

Here we describe a modified approach, which we call Tn5mCseq, that retains the advantages of transposase-based library preparation in the context of WGBS. Because the target of the transposition reaction is double-stranded DNA, whereas bisulfite treatment yields single-stranded DNA, the method was extensively modified such that the tagmentation reaction could take place prior to bisulfite treatment (Fig. 1B). First, the adaptors to be incorporated were methylated at all cytosine residues to maintain cytosine identity during bisulfite treatment, with the exception of the 19-bp transposase recognition sequence (in order to minimize differential binding during transposome assembly). Second, an oligonucleotide replacement scheme (Supplemental Fig. S1B; Grunenwald et al. 2011; Gertz et al. 2012) was utilized to ensure that each strand would have adaptors covalently attached to both ends of the molecule. Specifically, this entails initial transposition with a single adaptor in which the double-stranded transposase recognition sequence is truncated to $16 \mathrm{bp}\left(\mathrm{Tm}=36^{\circ} \mathrm{C}\right)$, thereby facilitating its post-incorporation removal by denaturation. A second adaptor is then annealed and the gap repaired, resulting in each strand being covalently flanked by both a $3^{\prime}$ and $5^{\prime}$ adaptor. The fragmented, adapted, double-stranded genomic DNA is then subjected to standard bisulfite treatment for the conversion of unmethylated cytosine to uracil. Degradation during the conversion process likely remains a primary source of loss, but the in- 
A

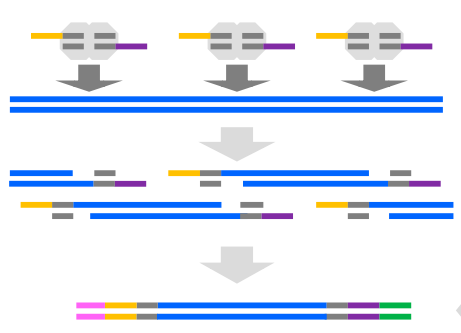

B
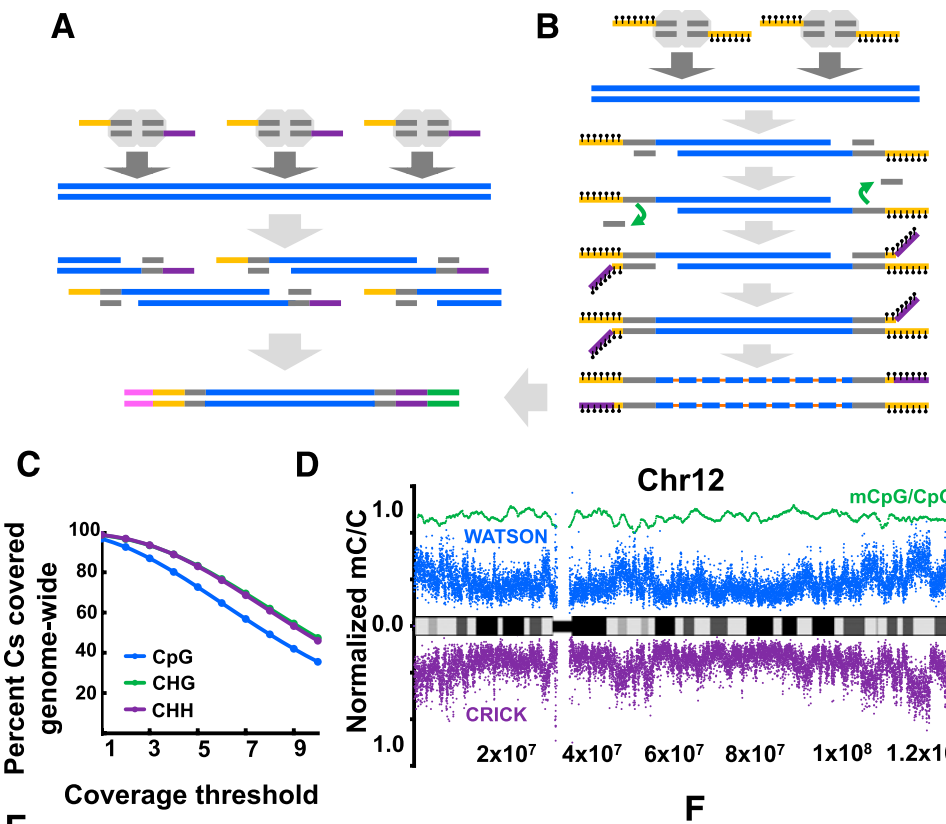

D

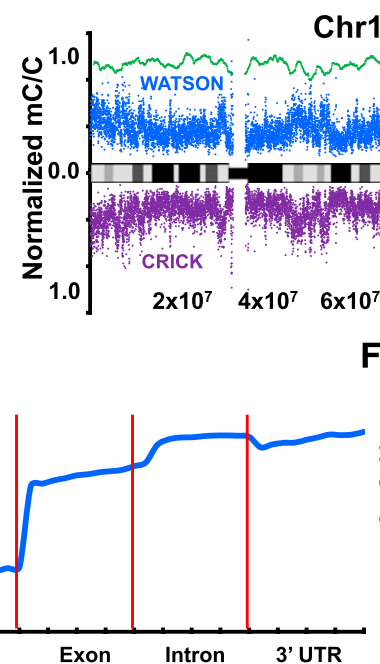

Chr12

E

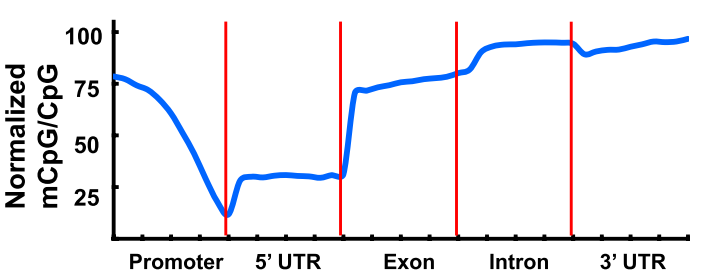

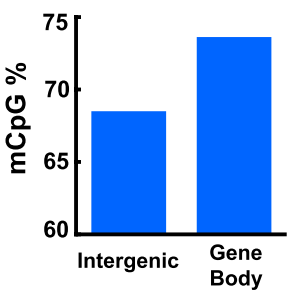

Figure 1. The Tn5mC-seq method and resulting methylation profiles. (A) Tagmentation-based DNAseq library construction. Genomic DNA is attacked by transposase homodimers loaded with synthetic, discontinuous oligos (yellow, purple) that allow for fragmentation and adaptor incorporation in a single step. Subsequent PCR appends outer flowcell-compatible primers (pink, green). (B) Tn5mC-seq library construction. Loaded transposase attacks genomic DNA with a single methylated adaptor (yellow). An oligo-replacement approach anneals a second methylated adaptor (purple), which is then subject to gap-repair. Bisulfite treatment then converts unmethylated cytosine to uracil (orange) followed by PCR to append outer flowcell-compatible primers (pink, green). Methylation is represented as black lollipops. (C) Coverage of cytosine positions genome-wide. More than $96 \%$ of $C s$ in all three contexts are covered at least once. Slight decrease in $\mathrm{CpG}$ coverage is due to reduced read alignment ability at regions with a high density of methylation. $(D)$ Normalized methylated cytosine over total cytosine positions in 10-kb windows across chromosome 12 (blue and purple, left axis), and normalized methylated $\mathrm{CpG}$ over total $\mathrm{CpG}$ in 100-kb windows across chromosome 12 (green, right axis). (E) Normalized methylated $\mathrm{CpG}$ over total $\mathrm{CpG}$ residues at annotated genic loci. Promoter is defined as 2-kb region upstream of TSS. ( $F$ ) Elevated CpG methylation levels in gene body (intron, exon) compared to intergenic regions.

creased efficiency of the prior steps and the lack of gel-based size selection result in an overall increase in the fraction of DNA that is converted, PCR-amplified, and sequenced.

\section{Results}

\section{Ultra-low-input transposase-based WGBS library performance}

We applied Tn5mC-seq to sequence the methylome of a lymphoblastoid cell line (GM20847) using libraries constructed from 1-200 ng of input genomic DNA. Each library was barcoded during PCR amplification and subjected to either a spike-in (5\%) or majority $(80 \%-90 \%)$ of a lane of sequencing on an Illumina HiSeq2000 (paired-end $100 \mathrm{bp}$ [PE100]; v2 chemistry with custom sequencing primers). These data are summarized in Table 1 and Supplemental Figure S2. In addition, several PCR conditions were investigated to

optimize amplification uniformity (Supplemental Fig. S3), as well as a modified protocol (Tn5mC-seq 1.1) (Supplemental Figs. S1D, S4) that eliminates the need for custom sequencing primers and may increase library construction efficiency. Reads were aligned to an in silico converted hg19 (GRC37) to both the top $(\mathrm{C} \rightarrow \mathrm{T})$ and bottom $(\mathrm{G} \rightarrow \mathrm{A})$ strands using BWA (Li and Durbin 2009) followed by read trimming of unmapped reads and secondary alignment using the same parameters. Unaligned reads typically consisted of low-quality artifacts that likely arose during amplification due to the reduced base complexity of bisulfite converted amplicons.

For each library constructed using $\geq 10$ ng of genomic DNA, over 100 million aligned reads were obtained (60\%-75\% of total filtered reads; see Methods) of high complexity $(90 \%-97 \%$ nonduplicates). Despite the significantly reduced performance of libraries prepared from $1 \mathrm{ng}, \sim 12$ million reads were still aligned and the library was of reasonable complexity (78\% nonduplicates). Post-alignment reads were merged and quality filtered for a total of $51.7 \mathrm{~Gb}$ of aligned, unique sequence. The average read depth was $8.6 \times$ per strand with $>96 \%$ of $\mathrm{CpG}$ and $>98 \%$ of non-CpG cytosines covered genome-wide (Fig. 1C; Supplemental Fig. S2). Because unmethylated nucleotides are incorporated during the gap-repair step (first $9 \mathrm{bp}$ of the second read and last $9 \mathrm{bp}$ before the adaptor as determined by insert size on the first read), the gaprepair regions must be excluded from methylation analysis. However, these bases also serve as an internal control for the conversion rate of the bisulfite treatment. We found this to be $>99 \%$ for all libraries, and this was independently confirmed using unmethylated lambda DNA spike-ins to two libraries.

For comparison, ligation chemistry-based libraries were constructed using 1000, 100, and 10 ng of GM20847 DNA of the same isolation as the batch used for Tn $5 \mathrm{mC}$-seq. These libraries were prepared following the protocols outlined by Lister et al. (2009) with the exception of PCR, which was performed using Kapa Robust due to its higher efficiency over other polymerase choices (Supplemental Fig. S3) During amplification, the 100 and 10 ng preparations did not show significant amplification above a negative control background and were not carried through to sequencing, precluding a comparison of $\mathrm{Tn} 5 \mathrm{mC}$-seq and ligation chemistrybased library construction with identical inputs (a $1000 \mathrm{ng}$ Tn5mCseq preparation was also not feasible due to the dilute concentrations of the commercially available transposase, which would result in a reduced density of transposition events on a high input mass).

Post-alignment, the $1000 \mathrm{ng}$ ligation chemistry-based library provided slightly more uniform coverage than Tn5mC-seq 1.1

\section{Genome Research} www.genome.org 
Table 1. Summary of $\mathrm{Tn} 5 \mathrm{mC}$-seq libraries and sequencing

\begin{tabular}{lrccrc}
\hline Name & $\begin{array}{c}\text { Input } \\
\text { DNA } \\
\text { (ng) }\end{array}$ & $\begin{array}{c}\text { Percentage } \\
\text { aligning }\end{array}$ & $\begin{array}{c}\text { Percentage } \\
\text { unique }\end{array}$ & $\begin{array}{c}\text { Unique } \\
\text { aligned } \\
\text { reads }\end{array}$ & $\begin{array}{c}\text { Mean } \\
\text { insert } \\
\text { size (bp) }\end{array}$ \\
\hline Tn5mC-C & 200 & 68 & 93 & $127,098,152$ & 198 \\
Tn5mC-D & 50 & 75 & 90 & $133,383,834$ & 254 \\
Tn5mC-E & 1 & 12 & 76 & $11,181,960$ & 134 \\
Tn5mC-F & 10 & 65 & 95 & $118,170,302$ & 168 \\
Tn5mC-G & 50 & 61 & 97 & $87,294,793$ & 180 \\
Tn5mC-H & 1 & 11 & 78 & $12,393,357$ & 126 \\
Tn5mC-1 & 10 & 62 & $\mathrm{n} / \mathrm{a}$ & $29,546,077$ & $\mathrm{n} / \mathrm{a}$ \\
Tn5mC-J & 50 & 71 & 95 & $132,144,644$ & 196 \\
TOTAL & & & & $651,213,119$ & \\
\hline
\end{tabular}

Raw reads were initially filtered for instrument valve failures at specific locations of reads and then removal of reads containing over three Ns or extremely low-quality bases (phred score $\leq 2$ ) in the first 50 bases. Alignment was then performed using BWA (Li and Durbin 2009) to in silico converted top and bottom strand references of hg19 (GRC37) followed by trimming and realignment. Duplicate reads were identified and removed according to their start position and insert size. The percentage of postfiltering reads that align for each library is shown, as is the percentage of these that are nonduplicates.

avalve failures in read 2 resulted in extensive read trimming (50-70 bp).

${ }^{\mathrm{b}}$ Complete valve failure on read 2 .

(Supplemental Fig. S1D) libraries constructed from 10 ng, particularly at the lower $\mathrm{CpG}$ densities that represent the majority of the genome (Supplemental Fig. S5A). Comparable uniformity was also observed with respect to $\mathrm{G}+\mathrm{C}$ content as well as for tetramer/ pentamer sequence contexts (Supplemental Fig. S5B,C). We also compared the methylation levels of CpGs well-covered by sequencing of libraries corresponding to both methods, and observed good agreement at positions with $5 \times$ or greater coverage $\left(\mathrm{r}^{2}=0.55\right)$ as well as $10 \times$ or greater coverage $\left(\mathrm{r}^{2}=0.82\right)$ (Supplemental Fig. S5D).

\section{Lymphoblastoid cell line methylation}

We were able to detect $\sim 46$ million $5 \mathrm{mC}$ positions (1\% FDR; see Methods), accounting for $4.2 \%$ of total cytosines with coverage. The majority of methylation observed was in the CpG context (97.1\%), and the global CpG methylation level was 69.1\%. This level is similar to that of the fetal fibroblast cell line IMR90 sequenced by Lister et al. $(2009 ; 67.7 \%)$ and is consistent with the observation that $\mathrm{CpG}$ methylation levels are reduced in differentiated cell types. Additionally, CHG and CHH methylation levels were substantially lower than in ES cells, at $0.36 \%$ and $0.37 \%$, respectively, again consistent with the differentiated cell type. On the chromosome scale, the methylation density correlated with banding patterns and increasing levels were observed extending distally through subtelomeric regions (Fig. 1D). An analysis of functionally annotated genic regions revealed a sharp decrease in CpG methylation through the promoter region followed by a minor increase in the 5' UTR and then elevated levels of methylation throughout the gene body, particularly at introns (Fig. 1E,F), consistent with previously described $\mathrm{CpG}$ methylation profiles (Lister et al. 2009).

\section{Discussion}

We developed $\mathrm{Tn} 5 \mathrm{mC}$-seq as a novel method for rapidly preparing complex, shotgun bisulfite sequencing libraries for WGBS. In brief, the method utilizes a hyperactive Tn5 transposase derivative to fragment genomic DNA and append adaptors in a single step, as previously characterized for the construction of DNA-seq libraries (Adey et al. 2010). In order for library molecules to withstand bisulfite treatment, the adaptors are methylated at all cytosine residues, and an oligonucleotide replacement strategy is employed to make each single-strand covalently flanked by adaptors. The high efficiency of the transposase and overall reduction in lossassociated steps permits construction of high-quality bisulfite sequencing libraries from as little as $10 \mathrm{ng}$ that are comparable to ligation chemistry-based libraries generated from $100 \times$ more DNA, as well as useful sequence from $1 \mathrm{ng}$ of input DNA. Additionally, the increased efficiency of transposase-mediated library construction may allow for preparation of WGBS libraries from poor-quality or degraded DNA samples.

Our results illustrate how derivatives of the transposase-based method for DNA-seq library preparation can enable key applications of next-generation sequencing where its advantages are perhaps even more relevant. The ability to generate such libraries from very low amounts of input genomic DNA substantially improves the practicality of whole methylome sequencing and removes a key advantage of less encompassing methods such as RRBS (Meissner et al. 2005; Harris et al. 2010). Specifically, low-input WGBS with Tn5mC-seq may make possible the comprehensive interrogation of methylation in many contexts where DNA quantity is a bottleneck, e.g., developing anatomical structures, microdissected tissues, or pathologies such as cancer, where the epigenetic landscape is of interest but tissue quantity limits highresolution WGBS.

\section{Methods}

\section{Tn5mC-seq library construction and sequencing}

Transposome complexes were generated by incubating $2.5 \mu \mathrm{L}$ of $10 \mu \mathrm{M}$ Tn5mC-A1 (Tn5mC-A1top: 5'-GAT [5mC] TA [5mC] A[5mC] $\mathrm{G}[5 \mathrm{mC}][5 \mathrm{mC}] \mathrm{T}[5 \mathrm{mC}][5 \mathrm{mC}][5 \mathrm{mC}] \mathrm{T}[5 \mathrm{mC}] \mathrm{G}[5 \mathrm{mC}] \mathrm{G}[5 \mathrm{mC}]$ [5mC] AT [5mC] AGAGATGTGTATAAGAGACAG-3', IDT, annealed to Tn5mC-A1bot: 5'-[Phos]-CTGTCTCTTATACACA-3', IDT, by incubating $10 \mu \mathrm{L}$ of each oligo at $100 \mu \mathrm{M}$ and $80 \mu \mathrm{L}$ of $\mathrm{EB}$ [QIAGen] for $2 \mathrm{~min}$ at $95^{\circ} \mathrm{C}$ and then cooling to room temperature at $0.1^{\circ} \mathrm{C} / \mathrm{sec}$ ) with $2.5 \mu \mathrm{L} 100 \%$ glycerol and $5 \mu \mathrm{L} \mathrm{Ez-Tn} 5$ transposase (Epicentre - Illumina) for $20 \mathrm{~min}$ at room temperature.

Genomic DNA prepared from GM20847 cell lines was used at respective input quantities with $4 \mu \mathrm{L}$ Nextera HMW Buffer (Epicentre-Illumina), nuclease-free water (Ambion) to $17.5 \mu \mathrm{L}$ and $2.5 \mu \mathrm{L}$ prepared $\mathrm{Tn} 5 \mathrm{mC}$ transposomes (regardless of the quantity of DNA used). Reactions were incubated for $9 \mathrm{~min}$ at $55^{\circ} \mathrm{C}$ in a thermocycler followed by SPRI bead cleanup (AMPure) using $36 \mu \mathrm{L}$ of beads and the recommended protocol with elution in $14 \mu \mathrm{L}$ nuclease-free water (Ambion). Adaptor 2 annealing was then carried out by adding $2 \mu \mathrm{L}$ of $10 \times$ Ampligase Reaction Buffer (EpicentreIllumina), $2 \mu \mathrm{L} 10 \times \mathrm{dNTPs}(2.5 \mathrm{mM}$ each; Invitrogen), and $2 \mu \mathrm{L}$ $10 \mu \mathrm{M}$ Tn5mC-A2top (5' -/5Phos/ CTGTCTCTTATACACATCT [5mC] TGAG [5mC] GGG [5mC] TGG [5mC] AAGG [5mC] AGA [5mC] [5mC] GAT [5mC]-3'; IDT) to each reaction and incubating for $2 \mathrm{~min}$ at $50^{\circ} \mathrm{C}$ followed by $10 \mathrm{~min}$ at $45^{\circ} \mathrm{C}$ and cooling at $0.1^{\circ} \mathrm{C} / \mathrm{sec}$ to $37^{\circ} \mathrm{C}$ and subsequent incubation for $10 \mathrm{~min}$. Gap-repair was then performed by adding $3 \mu \mathrm{L}$ of Ampligase at $5 \mathrm{U} / \mu \mathrm{L}$ (Epicentre-Illumina) and $1 \mu \mathrm{L}$ of either T4 DNA Polymerase (Tn5mC libraries A-G, NEB) or Sulfolobus DNA Polymerase IV (Tn5mC libraries H-J, NEB) and additional incubation for $30 \mathrm{~min}$ at $37^{\circ} \mathrm{C}$. Reactions were then cleaned up using SPRI beads (AMPure) according to recommended protocol using $36 \mu \mathrm{L}$ beads and elution in $50 \mu \mathrm{L}$ nuclease-free water (Ambion). Bisulfite treatment was performed using an EZ 
DNA Methylation Kit (Zymo) according to recommended protocols with a $14-\mathrm{h} 50^{\circ} \mathrm{C}$ incubation and $10 \mu \mathrm{L}$ elution. Eluate was then used as the template for PCR using $12.5 \mu \mathrm{L}$ Kapa 2G Robust HotStart ReadyMix (Kapa Biosystems), $1 \mu \mathrm{L} 10 \mu \mathrm{M}$ Tn5mC-P1 (5'AATGATACGGCGACCACCGAGATCTACACGCCTCCCTCGCG CCATCAG-3'; IDT), $1 \mu \mathrm{L} 10 \mu \mathrm{M}$ barcoded P2 (from Adey et al. 2010), $0.15 \mu \mathrm{L} 100 \times$ SYBR Green (Invitrogen), and $0.35 \mu \mathrm{L}$ nuclease-free water (Ambion). Thermocycling was carried out on a BioRad Opticon Mini real-time machine with the following parameters: 5 min at $95^{\circ} \mathrm{C}$; $\left(15 \mathrm{sec}\right.$ at $95^{\circ} \mathrm{C} ; 15 \mathrm{sec}$ at $62^{\circ} \mathrm{C} ; 40 \mathrm{sec}$ at $72^{\circ} \mathrm{C}$; Plate Read; $10 \mathrm{sec}$ at $\left.72^{\circ} \mathrm{C}\right) \times 99$. Reactions were monitored and removed from thermocycler as soon as plateau was reached (12-15 cycles).

Sequencing was carried out using either a full or partial lane on an Illumina HiSeq2000 using custom sequencing primers: read 1, Tn5mC-R1 (5' -GCCTCCCTCGCGCCATCAGAGATGTGTATA AGAGATAG-3'; IDT); index read, Tn5mC-Ix (5'-TTGTTTTTTATATA TATTTCTGAGCGGGCTGGCAAGGC-3'; IDT); and read 2, Tn5mC-R2 (5'-GCCTTGCCAGCCCGCTCAGAAATATATATAAAAAACAA-3'; IDT). Read lengths were either single-read at 36 bp with a 9-bp index (SE36, libraries A and B, not included in Table 1) or 101 bp pairedend with a 9-bp index (PE101, libraries C-J). Libraries were only sequenced on runs that did not have lanes containing Nextera libraries as a precaution due to the similarity between sequencing primers.

Tn5mC-Seq 1.1 library preparation (Supplemental Fig. 1D) was carried out as previously described with several modifications: (1) Transposase recognition sequence reverse compliment is $3^{\prime}$ blocked to prevent nonspecific extension in final PCR. (2) Replacement oligo is methylated through the region complementary to the transposase recognition sequence to maintain complexity during bisulfite conversion and allow the use of standard Nextera sequencing primers. (3) Replacement oligo is 3' blocked to prevent degradation by $3^{\prime} \rightarrow 5^{\prime}$ exonuclease activity of gap-repair polymerase (replacement oligo: Tn5mC1.1-A2top 5'-/5Phos/ [5mC] TGT [5mC] T [5mC] TTATA [5mC] A [5mC] AT [5mC] T [5mC] TGAG [5mC] GGG [5mC] TGG [5mC] AAGG [5mC] AGA [5mC] [5mC] GA [inv dT]-3', IDT; blocked transposase recognition sequence end: Tn5mC1.1-A1bot3block 5'-[Phos]-CTGTCTCTTA TACA [ddC]-3'). Duplicate libraries were prepared from $100 \mathrm{ng}$, $10 \mathrm{ng}$, and $1 \mathrm{ng}$ of starting material and were subject to PCR amplification using either Kapa HiFi U+ Hot Start Ready Mix, or Kapa 2G Robust Hot Start Ready Mix (Kapa Biosystems) and were sequenced on a single-end 36-bp read plus a 9-bp index read run on an Illumina GAIIx. Library characterization can be found in Supplemental Figure 5.

\section{Ligation chemistry WGBS library construction and sequencing}

We subjected 1000, 100, and 10 ng of genomic DNA prepared from GM18507 cell lines to ligation chemistry-based library preparation according to methods described by Lister et al. (2009) with several minor exceptions: (1) Bisulfite conversion was carried out using an EZ DNA Methylation Kit (Zymo), and (2) PCR was carried out using Kapa 2G Robust Hot Start Ready Mix (Kapa Biosystems). The change in PCR enzyme was due to several unpublished experiments demonstrating a much higher efficiency with Kapa Robust as opposed to PfuTurbo Cx used according to the method described by Lister et. al.(2009). Sequencing was performed on an Illumina MiSeq instrument using a single-end 100-bp sequence read run.

\section{Read filtering and alignment}

The hg19 reference genome was first bisulfite-converted in silico for both the top ( $\mathrm{C}$ changed to T, C2T) and bottom ( $\mathrm{G}$ changed to
A, G2A) strands. Prior to alignment, reads were filtered based on the run metrics, as several libraries were run on lanes in which instrument valve failures resulted in poor quality or reads consisting primarily of $\mathrm{N}$ bases. Filtering was carried out by first calculating the base compositions as well as mean base quality scores at each position in the read. Many of the lanes had significantly reduced quality scores at the start and/or end of the read and were globally trimmed to remove any start or end positions that had a mean phed score of less than or equal to 10 . The start and ends of the reads were additionally globally trimmed if a position within the first or last 25 bases of the read had a mean composition of $10 \%$ Ns, which generally corresponded to the quality-based trimming. Additionally, reads that contained three or more Ns were also removed. It is important to note that the reduced qualities in the runs were "flowcell-wide" regardless of the library that was run and not isolated to Tn5mC-seq libraries. Subsequent runs for the Tn5mC-seq 1.1 and polymerase testing experiments did not suffer instrument failures, and no trimming of the reads was necessary. Next, reads were aligned to both the C2T and G2A strands using BWA with default parameters. Reads that aligned to both strands were removed. Read pairs in which neither aligned to either strand were then pulled and trimmed to $76 \mathrm{bp}$ (except for SE36 runs) and again aligned to both C2T and G2A strands. Duplicate reads (pairs sharing the same start positions for both reads 1 and 2) were removed and complexity determined. Reads with an alignment score less than 10 were then filtered out prior to secondary analysis. Total fold coverage was calculated using the total bases aligned from unique reads over the total alignable bases of the genome (slightly below $3 \mathrm{~Gb}$ per strand).

\section{$5 \mathrm{mC}$ calling}

Methylated cytosines were called using a binomial distribution as in the method described by Lister et al. (2009), whereby a probability mass function is calculated for each methylation context ( $\mathrm{CpG}, \mathrm{CHG}, \mathrm{CHH}$ ) using the number of reads covering the position as the number of trials and reads maintaining cytosine status as successes with a probability of success based on the total error rates that were determined by the combined nonconversion rate and sequencing error rate. The total error rate was initially determined by unmethylated lambda DNA spike-ins; however, we found that the error rate estimation from the gap-repair portion of reads (as described in the main text) gave a more comprehensive estimate, which was slightly higher than that of the lambda estimate. Therefore to be conservative, we used the highest determined error rate at 0.009 . If the probability was below the value of $M$, where $\mathrm{M} \times$ (number of total unmethylated $\mathrm{CpG})<0.01 \times($ number of total methylated $\mathrm{CpG}$ ), the position was called as being methylated, thus enforcing that no more than $1 \%$ of positions would be due to the error rate.

\section{Data access}

The sequence data presented in this study have been submitted to the NCBI Sequence Read Archive (SRA) (http://www.ncbi.nlm. nih.gov/sra) under accession no. SRP011746.

\section{Acknowledgments}

We thank Cholie (Charlie) Lee for performing all sequencing runs and the Shendure laboratory for helpful discussions. We also thank Nick Caruccio, Haiying Gruenwald, Brad Baas, and Igor Goryshin from Epicentre (Illumina) for help and ideas regarding transposasebased library preparation, as well as Eric Van Der Walt and colleagues at Kapa Biosystems for early access to reagents and pro-

\section{Genome Research \\ www.genome.org}


tocols for library amplification. A.A. is funded by an NSF Graduate Research Fellowship. This work was supported in part by the Lowell Milken Prostate Cancer Foundation Young Investigator Award (J.S.). Author contributions: A.A. performed experiments and data analysis. A.A. and J.S. designed experiments and wrote the manuscript.

\section{References}

Adey A, Morrison HG, Asan, Xun X, Kitzman JO, Turner EH, Stackhouse B, MacKenzie AP, Caruccio NC, Zhang X, et al. 2010. Rapid, low-input, low-bias construction of shotgun fragment libraries by high-density in vitro transposition. Genome Biol 11: R119. doi: 10.1186/gb-2010-1112-r119.

Ball MP, Li JB, Gao Y, Lee JH, LeProust EM, Park IH, Xie B, Daley GQ, Church GM. 2009. Targeted and genome-scale strategies reveal gene-body methylation signatures in human cells. Nat Biotechnol 27: 361-368.

Clark SJ, Harrison J, Paul CL, Frommer M. 1994. High sensitivity mapping of methylated cytosines. Nucleic Acids Res 22: 2990-2997.

Cokus SJ, Feng S, Zhang X, Chen Z, Merriman B, Haudenschild CD, Pradhan S, Nelson SF, Pellegrini M, Jacobsen SE. 2008. Shotgun bisulphite sequencing of the Arabidopsis genome reveals DNA methylation patterning. Nature 452: 215-219.

Deng J, Shoemaker R, Xie B, Gore A, LeProust EM, Antosiewicz-Bourget J, Egli D, Maherali N, Park IH, Yu J, et al. 2009. Targeted bisulfite sequencing reveals changes in DNA methylation associated with nuclear reprogramming. Nat Biotechnol 27: 353-360.

Down TA, Rakyan VK, Turner DJ, Flicek P, Li H, Kulesha E, Graf S, Johnson N, Herrero J, Tomazou EM, et al. 2008. A Bayesian deconvolution strategy for immunoprecipitation-based DNA methylome analysis. Nat Biotechnol 26: 779-785.
Gertz J, Varley KE, Davis NS, Baas BJ, Goryshin IY, Vaidyanathan R, Kuersten S, Myers RM. 2012. Transposase mediated construction of RNA-seq libraries. Genome Res 22: 134-141.

Grunenwald H, Baas B, Goryshin I, Zhang B, Adey A, Hu S, Shendure J, Caruccio N, Maffitt M. 2011. Nextera PCR-free DNA library preparation for next-generation sequencing. (Poster presentation, AGBT 2011).

Gu H, Smith ZD, Bock C, Boyle P, Gnirke A, Meissner A. 2011. Preparation of reduced representation bisulfite sequencing libraries for genome-scale DNA methylation profiling. Nat Protoc 6: 468-481.

Harris RA, Wang T, Coarfa C, Nagarajan RP, Hong C, Downey SL, Johnson BE, Fouse SD, Delaney A, Zhao Y, et al. 2010. Comparison of sequencingbased methods to profile DNA methylation and identification of monoallelic epigenetic modifications. Nat Biotechnol 28: 1097-1105.

Li H, Durbin R. 2009. Fast and accurate short read alignment with BurrowsWheeler transform. Bioinformatics 25: 1754-1760.

Li Y, Zhu J, Tian G, Li N, Li Q, Ye M, Zheng H, Yu J, Wu H, Sun J, et al. 2010. The DNA methylome of human peripheral blood mononuclear cells. PLoS Biol 8: e1000533. doi: 10.1371/journal.pbio.1000533.

Lister R, Pelizzola M, Dowen RH, Hawkins RD, Hon G, Tonti-Filippini J, Nery JR, Lee L, Ye Z, Ngo QM, et al. 2009. Human DNA methylomes at base resolution show widespread epigenomic differences. Nature 462: 315-322.

Meissner A, Gnirke A, Bell GW, Ramsahoye B, Lander ES, Jaenisch R. 2005 Reduced representation bisulfite sequencing for comparative highresolution DNA methylation analysis. Nucleic Acids Res 33: 5868-5877.

Zilberman D, Henikoff S. 2007. Genome-wide analysis of DNA methylation patterns. Development 134: 3959-3965.

Ziller MJ, Muller F, Liao J, Zhang Y, Gu H, Bock C, Boyle P, Epstein CB, Bernstein BE, Lengauer T, et al. 2011. Genomic distribution and intersample variation of non-CpG methylation across human cell types. PLoS Genet 7: e1002389. doi: 10.1371/journal.pgen.1002389.

Received December 11, 2011; accepted in revised form March 29, 2012. 


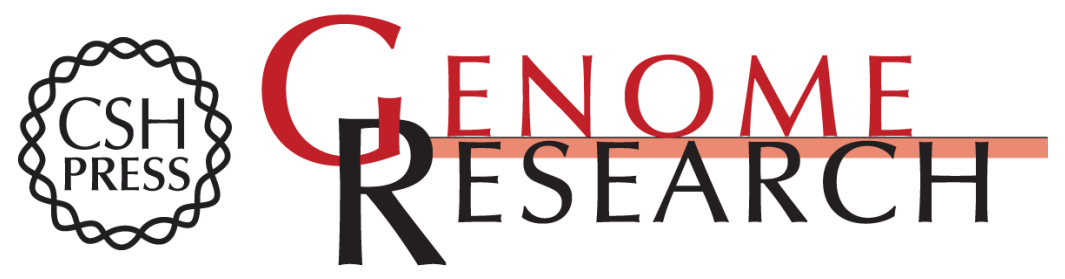

\section{Ultra-low-input, tagmentation-based whole-genome bisulfite sequencing}

Andrew Adey and Jay Shendure

Genome Res. 2012 22: 1139-1143 originally published online March 30, 2012

Access the most recent version at doi:10.1101/gr.136242.111

Supplemental Material

References

Creative

Commons

License

Email Alerting

Service
http://genome.cshlp.org/content/suppl/2012/03/30/gr.136242.111.DC1

This article cites 15 articles, 2 of which can be accessed free at: http://genome.cshlp.org/content/22/6/1139.full.html\#ref-list-1

This article is distributed exclusively by Cold Spring Harbor Laboratory Press for the first six months after the full-issue publication date (see

$\mathrm{http}: / / g$ enome.cshlp.org/site/misc/terms.xhtml). After six months, it is available under a Creative Commons License (Attribution-NonCommercial 3.0 Unported License), as described at http://creativecommons.org/licenses/by-nc/3.0/.

Receive free email alerts when new articles cite this article - sign up in the box at the top right corner of the article or click here.

\section{Affordable, Accurate Sequencing.}

To subscribe to Genome Research go to:

https://genome.cshlp.org/subscriptions 